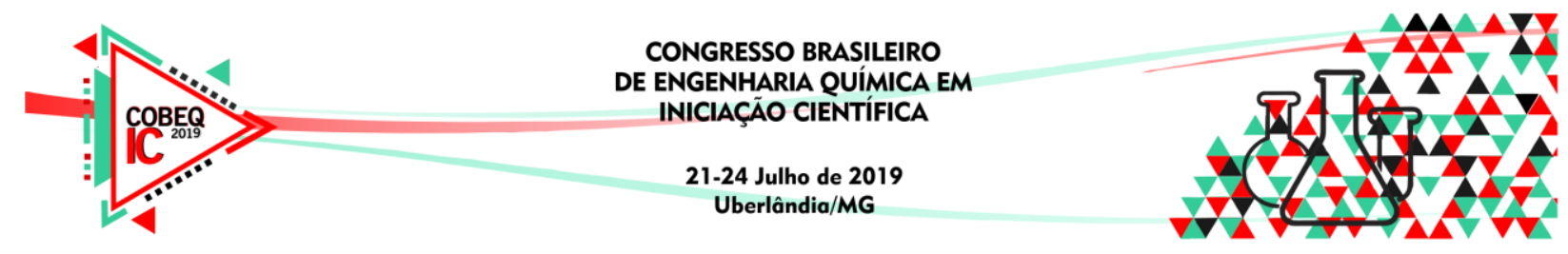

\title{
ESTUDO DAS MOLÉCULAS MAJORITÁRIAS DO EXTRATO DA CASCA DE CINNAMOMUM VERUM POR MODELAGEM MOLECULAR
}

\author{
A. L. S. CARVALHO ${ }^{1}$, E. MOCHIUTTI ${ }^{1}$, A. E. S. NASCIMENTO ${ }^{1}$, D. S. B. BRASIL ${ }^{1}$, M. \\ C. MARTELLI ${ }^{1}$ \\ ${ }^{1}$ Universidade Federal do Pará, Faculdade de Engenharia Química \\ E-mail para contato: mrcarvalhoarthur@gmail.com
}

\begin{abstract}
RESUMO - Produtos naturais podem possuir inúmeras propriedades biológicas benéficas para o organismo humano em diversos aspectos. $\mathrm{O}$ trabalho teve como objetivo utilizar de técnicas de modelagem molecular para indicar essas propriedades nas estruturas majoritárias do extrato de casca de Cinnamomum Verum e estabelecer se as mesmas podem ter alguma aplicação na indústria farmacêutica. Esse estudo teve como base o desenho das estruturas anteriormente isoladas e a aplicação de banco de dados PreAdmet para obter propriedades farmacocinéticas e toxicológicas desses compostos, além de uma análise físico-química destes compostos com o software Spartan. Como resultados, todas as moléculas demonstraram possibilidade de serem drogas de consumo oral por serem aprovadas da regra de Lipinski, além de possuírem boa capacidade de absorção no intestino. Porém, as mesmas apresentaram dificuldade de fixação nas células do organismo e duas estruturas (4-Vinylbenzoic acid e trans-Cinnamaldehyde) expressaram tendências cancerígenas em ratos e camundongos.
\end{abstract}

\section{INTRODUÇÃO}

A espécie Cinnamomum Verum (canela) pertence à família Lauraceae, é bastante utilizada em medicinas tradicionais, a casca é usada como condimento e agente aromatizante, sendo picante, amarga, aromática, adstringente e desodorizante. Além dos fatores sensoriais, essa espécie traz propriedades medicinais contra bronquite, asma, cefaléia (dor de cabeça), odontalgia (dor nos dentes), doenças cardíacas, entre outras. Por fim, ela também possui utilidade para ações antioxidantes, antifúngicas e anti-inflamatórias. As moléculas mais presentes no extrato da casca da canela são: (E)-3-(2-Methoxyphenyl)-2- propenoic acid (40,41\%), trans-Cinnamaldehyde (20,28\%), 4-Vinyl benzoic acid (10,54\%), coumarin (8,47\%). (MARIAPPAN et al., 2013).

Os produtos naturais vêm sendo usados na medicina tradicional durante toda a história da humanidade. Incluindo seus derivados, produtos naturais são cerca de um terço das $N M E$ (New Molecular Entities) aprovadas pela FDA (U.S. Food and Drug Administration). Alguns desafios são vinculados à produção de novas drogas utilizando esse tipo de matéria prima, como problemas com absorção, distribuição, metabolismo e excreção (ADME) no organismo para com essas estruturas (PRESS et al., 2019) 


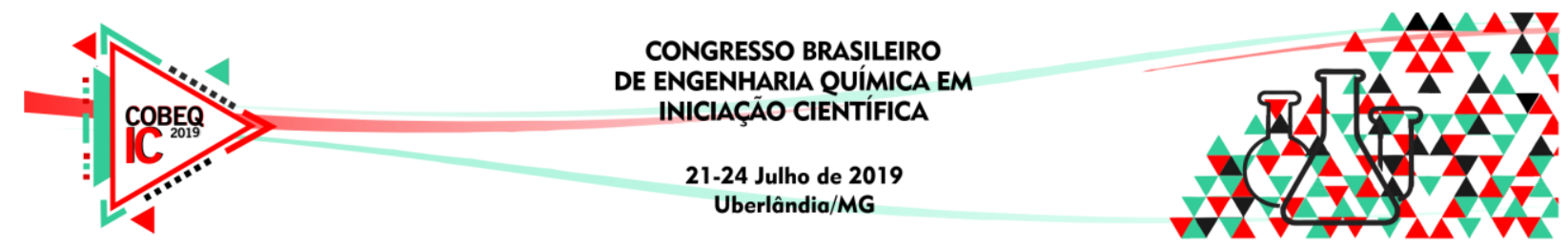

Neste trabalho, foram utilizadas técnicas de modelagem molecular para fazer uma predição de propriedades farmacocinéticas, quimiométricas e toxicológicas das moléculas majoritárias do extrato de casca de Cinnamomum Verum (canela) e indicar possíveis utilidades na indústria farmacêutica e problemas vinculados a essas estruturas.

\section{MATERIAIS E MÉTODOS}

As estruturas analisadas nesse trabalho foram selecionadas a partir do estudo do extrato da casca de Cinnamomum Verum feito por Mariappan (2013) e dispostas na Tabela 1, mostrada abaixo. Após a seleção, essas moléculas foram desenhadas bidimensionalmente com o auxílio do software ChemSketch. Em seguida, a geometria foi otimizada para um modelo tridimensional pelo software Avogadro utilizando o campo de força MMF94s, com o algoritmo steepest descent com 10000 passos.

Tabela 1 - Moléculas majoritárias da Cinnamomum Verum

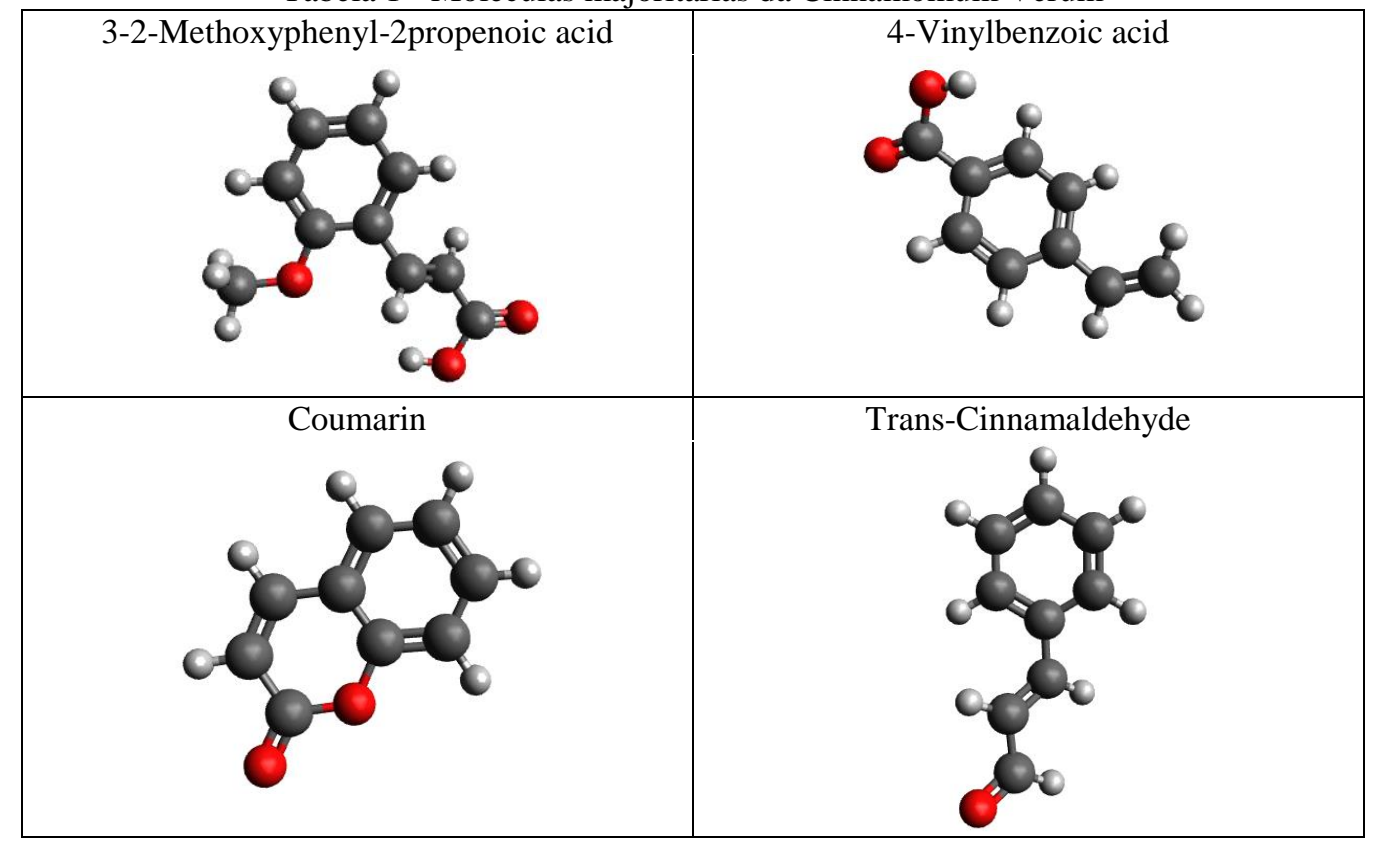

A minimização de energia destes compostos foi calculada pelo software Gaussian a partir da metodologia DFT (Density Functional Theory), utilizando a função B3LYP com base 6-31G* para uma aproximação de energia. Para confirmar a validade das estruturas obtidas e certificar que não exista frequência imaginária foram realizados os cálculos de frequências, pois, se ocorrer frequência imaginária, a estrutura não está no seu mínimo verdadeiro. (DAPPRICH et al., 1999).

As moléculas foram submetidas ao cálculo de propriedades físico-químicas no software Spartan. Assim destacando os parâmetros de maior interesse: Energia HOMO (energia do maior orbital preenchido), Energia LUMO (energia do menor orbital não preenchido), GAP 


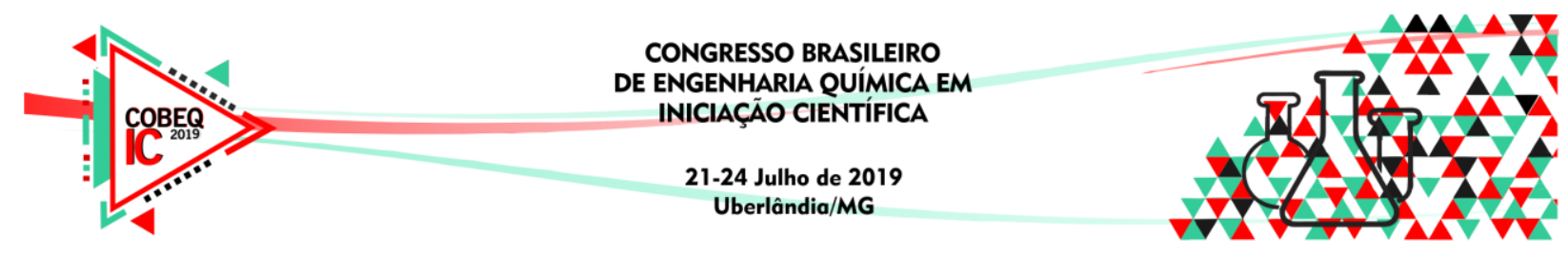

(diferença entre HOMO e LUMO), MWT (massa molar), PSA (área de superfície polar), LogP (lipossolubilidade), HBD e HBA (indicadores de ligações de hidrogênio).

Em $90 \%$ dos fármacos oralmente ativos, os compostos seguem um padrão chamado "regra dos 5" (Rule Of Five - RO5), em que 4 parâmetros estão em uma faixa de aceitação relacionados à solubilidade em água e permeabilidade intestinal. Os valores destas propriedades são restritos à MWT $\leq 500$ Dalton $(\mathrm{g} / \mathrm{mol}), \log \mathrm{P} \leq 5, \mathrm{HBD}($ Hydrogen Bond Donors $) \leq 5$, HBA (Hydrogen Bond Acceptors) $\leq 10$. Passar na RO5 não significa que o composto terá necessariamente utilidade como droga, afinal, a regra não especifica fatores estruturais destas substâncias. (LIPINSKI, 2004).

As moléculas foram ainda comparadas às do banco de dados PreAdmet para a obtenção dos resultados de ADME (Absorção, Distribuição, Metabolismo e Excreção): BBB, Caco2, HIA e PPB. E de Toxicidade: Mutagenicidade, Carcinogenicidade (ratos) e Carcinogenicidade (camundongos).

\section{RESULTADOS E DISCUSSÃO}

A tabela 2 apresenta resultados de algumas características físico-químicas relevantes para esse estudo.

Tabela 2 - Resultados de Propriedades Físico-Químicas obtidas pelo Spartan.

\begin{tabular}{|c|c|c|c|c|c|c|c|c|}
\hline & $\begin{array}{c}\text { HOMO } \\
(\mathrm{eV})\end{array}$ & $\begin{array}{c}\text { LUMO } \\
(\mathrm{eV})\end{array}$ & $\begin{array}{c}\text { GAP } \\
(\mathrm{eV})\end{array}$ & $\begin{array}{c}\text { MWT } \\
(\mathrm{Da})\end{array}$ & $\begin{array}{c}\text { PSA } \\
\left(\AA^{2}\right)\end{array}$ & HBD & HBA & $\operatorname{LogP}$ \\
\hline \hline $\begin{array}{c}\text { 3-2-Methoxyphenyl-2 } \\
\text { propenoic acid }\end{array}$ & $-6,21$ & $-1,84$ & 4,37 & 178,187 & 38,972 & 1 & 2 & 0,33 \\
\hline 4-Vinylbenzoic acid & $-6,59$ & $-1,81$ & 4,78 & 148,161 & 32,805 & 1 & 1 & 1,12 \\
\hline Coumarin & $-6,5$ & $-1,88$ & 4,62 & 146,145 & 21,371 & 0 & 1 & 0,51 \\
\hline Trans-Cinnamaldehyde & $-6,58$ & $-2,09$ & 4,49 & 132,162 & 14,942 & 0 & 1 & 1,04 \\
\hline
\end{tabular}

Maiores valores de Energia HOMO e Energia LUMO acusam uma maior tendência a doar elétrons e a receber elétrons respectivamente. Com relação ao GAP, quanto menor o número, mais a molécula se torna polarizável e reativa. (SIVAJEYANTHI et al., 2017). Desta forma, o trans-Cinnamaldehyde e o 4-Vinylbenzoic acid têm mais possibilidades de serem doadores de elétrons. Assim como o mesmo trans-Cinnamaldehyde se mostra como um bom receptor de elétrons. O GAP HOMO-LUMO tem como maior resultado o 4,78 do 4Vinylbenzoic acid, indicando este como o composto menos reativo. Diante disso, possivelmente, a molécula mais estável é 4-Vinylbenzoic acid e a mais instável é 3-2-Methoxyphenyl2 propenoic acid.

A área de superfície polar (PSA) está relacionada com a capacidade de absorção que o intestino terá quando em contato com estas estruturas: para PSA $<63 \AA^{2}$ a estrutura será completamente absorvida (HIA > 90\%), enquanto compostos com PSA $>139 \AA^{2}$, praticamente não serão absorvidos (HIA < 10\%). (PALM et al., 1997). Assim todas as estruturas possuem boa absorção quando sendo utilizadas como drogas de consumo oral. 


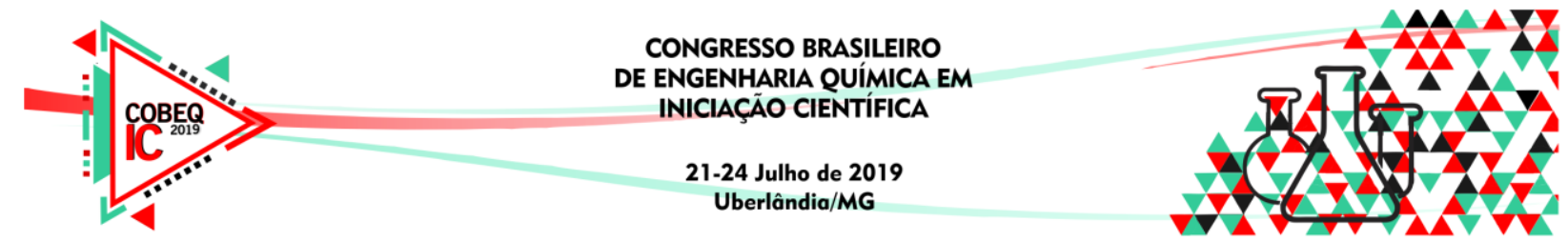

Os resultados das 4 propriedades abordadas pela RO5 (MWT, HBD, HBA e LogP) mostraram que todos os compostos seguem as normas propostas por Lipinski. Tendo MWT um valor menor que $500 \mathrm{Da}, \mathrm{HBD}$ e LogP menores que 5 e HBA sendo menor que 10 . O que significa que os compostos possuem propriedades dentro dos parâmetros estabelecidos para serem usados como drogas orais.

A tabela 3 mostra as propriedades de ADME dos compostos.

Tabela 3 - Resultados de ADME obtidos pelo banco de dados PreADME/T.

\begin{tabular}{|c|c|c|c|c|}
\hline Molécula & BBB & $\begin{array}{c}\text { Caco2 } \\
(\mathrm{nm} / \mathrm{seg})\end{array}$ & $\begin{array}{c}\text { HIA } \\
(\%)\end{array}$ & $\begin{array}{c}\text { PPB } \\
(\%)\end{array}$ \\
\hline \hline 3-2-Methoxyphenyl-2 propenoic acid & 1,74859 & 21,1225 & 96,72931 & 82,80881 \\
\hline 4-Vinylbenzoic acid & 1,89161 & 21,0421 & 97,8452 & 68,46717 \\
\hline Coumarin & 1,56844 & 32,1202 & 100 & 43,39511 \\
\hline trans-Cinnamaldehyde & 1,26197 & 23,784 & 100 & 52,89236 \\
\hline
\end{tabular}

Para os resultados de BBB (índice de penetração na barreira hematoencefálica), segundo a escala proposta por Ma (2005), as 4 moléculas possuem esse índice de forma moderada, já que se encontram entre 0,1 e 2 . Com base nesses resultados, o estudo não especifica se as estruturas estudadas podem ser danosas ao sistema nervoso, ou seja, faz-se necessária uma análise mais aprofundada dos efeitos dessas moléculas para julgar se esse índice é o ideal para tais substâncias.

Com relação a Caco2, o PreAdmet utiliza uma análise feita por Yamashita (2000), em que menos de $4 \mathrm{~nm} / \mathrm{seg}$ indica baixa permeabilidade em células intestinais, entre 4 e 70 $\mathrm{nm} / \mathrm{seg}$, uma permeabilidade mediana e mais de $70 \mathrm{~nm} / \mathrm{seg}$ uma alta permeabilidade. Dessa forma, todos os compostos estão na faixa de permeabilidade moderada, indicando que não demorariam a ser absorvidos pelo intestino delgado.

Quanto aos valores de HIA, todas as substâncias obtiveram resultados acima de 95\%, sendo que, as moléculas Coumarin e trans-Cinnanmaldehyde apresentaram 100\% de absorção. Esse parâmetro está relacionado à capacidade total de absorção pelo intestino.

Os dados de PPB (Plasma Protein Binding) indicam a capacidade de se fixar em proteínas das células do organismo. A escala utilizada indica que qualquer resultado menor que 90\% (como o das moléculas estudadas) traduz uma dificuldade de fixação, e, consequentemente, uma menor capacidade de distribuição pelo corpo. 


\begin{tabular}{|c|c|c|c|}
\hline \multicolumn{4}{|c|}{$\begin{array}{l}\text { CONGRESSO BRASILEIRO } \\
\text { DE ENGENHARIA QUÍMICA EM } \\
\text { INICIAÇÃOO CIENTIIFICA }\end{array}$} \\
\hline \multicolumn{4}{|c|}{$\begin{array}{l}\text { Os parâmetros da tabela } 4 \text { avaliam a toxicidade das substâncias em questão no } \\
\text { nano. }\end{array}$} \\
\hline Molécula & Mutagenicidade & $\begin{array}{l}\text { Carcinogenicidade } \\
\text { (Ratos) }\end{array}$ & $\begin{array}{l}\text { Carcinogenicidade } \\
\text { (Camundongos) }\end{array}$ \\
\hline 3-2-Methoxyphenyl-2 propenoic acid & Mutagênico & Não & Não \\
\hline 4-Vinylbenzoic acid & Mutagênico & Sim & Sim \\
\hline Coumarin & Mutagênico & Não & Não \\
\hline Trans-Cinnamaldehyde & Mutagênico & Sim & Sim \\
\hline
\end{tabular}

A princípio, nota-se que todas apresentaram características mutagênicas. O que permite várias interpretações sobre o efeito (negativo ou positivo) delas em nível celular. A partir do resultado positivo de mutagenicidade não se sabe se a molécula é de fato prejudicial para humanos, haja vista que não há um padrão de predição para relacionar esse fator com a carcinogenicidade. Sendo assim, o banco de dados permite uma predição de carcinogenicidade para ratos e camundongos. Dessa forma, se o composto apresenta uma tendência cancerígena para estas espécies, pode indicar uma maior probabilidade dessa mesma tendência ser aplicada para seres humanos. Os resultados mostraram que duas moléculas foram consideradas cancerígenas para ambas as espécies (4-Vinylbenzoic acid e trans-Cinnamaldehyde) e duas obtiveram os resultados opostos (3-2-Methoxyphenyl-2 propenoic acid e Coumarin).

\section{CONCLUSÃO}

Como conclusão para o estudo realizado, as estruturas em questão demonstraram uma possibilidade em serem drogas oralmente ativas devido aos seus resultados referentes à regra de Lipinski (características físico-químicas favoráveis). Em que as mesmas não violaram a regra. Além disso, ao analisar as propriedades farmacocinéticas, foi observado que as 4 substâncias obtiveram resultados em faixas semelhantes em todos os parâmetros. Porém, com relação às propriedades toxicológicas, duas moléculas se mostraram não cancerígenas para ratos e camundongos (apesar de todas apresentarem tendência mutagênica). Assim, conclui-se que essas duas estruturas (3-2-Methoxyphenyl-2 propenoic acid e Coumarin) são as mais promissoras em um âmbito farmacológico, sendo assim necessários estudos mais profundos para saber todos os seus efeitos no organismo.

\section{REFERÊNCIAS}

DAPPRICH, S.; KOMÁROMI, I.; BYUN, K. S.; MOROKUMA, K.; FRISCH, M. J. A new ONIOM implementation in Gaussian98. Part I. The calculation of energies, gradients, vibrational frequencies and electric field derivatives. Journal of Molecular Structure: THEOCHEM, p. 461-462, 1999.

LIPINSKI, C. A. Lead- and drug-like compounds: The rule-of-five revolution. Drug Discovery Today: Technologies, v. 1, n. 4, p. 337-341, 2004. 


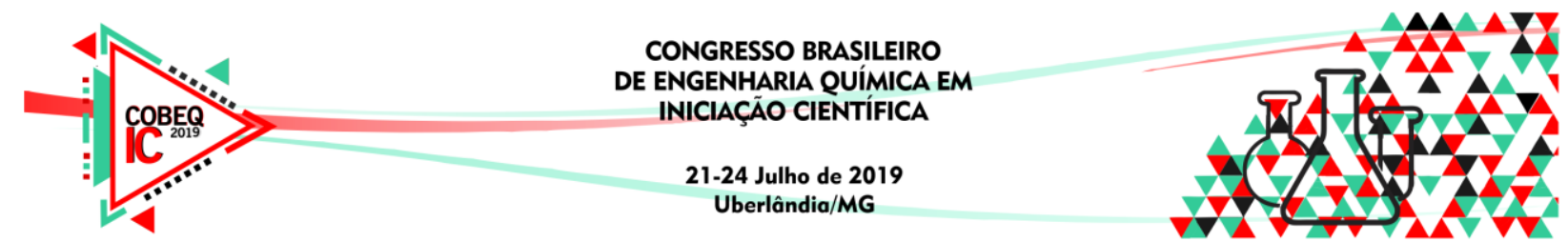

MA, X.; CHEN, C.; YANG, J. Predictive model of blood-brain barrier penetration of organic compounds. Acta Pharmacologica Sinica, v. 26, p. 500-512, 2005.

MARIAPPAN, P. M.; SABESAN, G.; KOILPILLAI, B.; JANAKIRAMAN, S.; SHARMA, N. K. Chemical characterisation and antifungal activity of methanolic extract of Cinnamomum verum J. Presl bark against Malassezia spp. Pharmacognosy Journal, v. 5, n. 5, p. 197-204, 2013.

PALM, K.; STENBERG, P.; LUTHMAN, K.; ARTURSSON, P. Polar molecular surface properties predict the intestinal absorption of drugs in humans. Pharmaceutical Research, v. 14, n. 5, p. 568-571, 1997.

PRESS, N. J.; JOLY, E.; ERTL, P. Natural product drug delivery: A special challenge? Progress in Medicinal Chemistry, p. 2, 2019.

SIVAJEYANTHI, P.; JEEVARAJ, M.; BALASUBRAMANI，K.; VISWANATHAN, V.; VELMURUGAN, D. Synthesis, crystal structure, Hirshfeld surface analysis and frontier molecular orbital analysis of 2-((2-hydroxynaphthalen-1yl)methylene)hydrazinecarbothioamide. Chemical Data Collections, v. 11-12, p. 220$231,2017$.

YAMASHITA, S.; FURUBAYASHI, T.; KATAOKA, M.; SAKANE, T.; SEZAKI, H.; TOKUDA, H. Optimized conditions for prediction of intestinal drug permeability using Caco-2 cells. Eur. J. Pharm. Sci, v. 10, p. 195-204, 2000. 